\title{
Ray Tracing Study of Optical Characteristics of the Solar Image in the Receiver for a Thermal Solar Parabolic Dish Collector
}

\author{
Saša R. Pavlovic and Velimir P. Stefanovic \\ Faculty of Mechanical Engineering, Department of Energetics and Process Technique, University of Niš, Niš, Serbia \\ Correspondence should be addressed to Saša R. Pavlovic; saledoca@gmail.com
}

Received 15 June 2015; Revised 2 September 2015; Accepted 27 September 2015

Academic Editor: Santanu Bandyopadhyay

Copyright ( 92015 S. R. Pavlovic and V. P. Stefanovic. This is an open access article distributed under the Creative Commons Attribution License, which permits unrestricted use, distribution, and reproduction in any medium, provided the original work is properly cited.

\begin{abstract}
This study presents the geometric aspects of the focal image for a solar parabolic concentrator (SPC) using the ray tracing technique to establish parameters that allow the designation of the most suitable geometry for coupling the SPC to absorber-receiver. The efficient conversion of solar radiation into heat at these temperature levels requires a use of concentrating solar collectors. In this paper detailed optical design of the solar parabolic dish concentrator is presented. The system has diameter $D=3800 \mathrm{~mm}$ and focal distance $f=2260 \mathrm{~mm}$. The parabolic dish of the solar system consists of 11 curvilinear trapezoidal reflective petals. For the construction of the solar collectors, mild steel-sheet and square pipe were used as the shell support for the reflecting surfaces. This paper presents optical simulations of the parabolic solar concentrator unit using the ray tracing software TracePro. The total flux on the receiver and the distribution of irradiance for absorbing flux on center and periphery receiver are given. The goal of this paper is to present the optical design of a low-tech solar concentrator that can be used as a potentially low cost tool for laboratory scale research on the medium-temperature thermal processes, cooling, industrial processes, polygeneration systems, and so forth.
\end{abstract}

\section{Introduction and Survey of Literature}

This paper presents the numerical results of the optimization of the solar image in a receiver for a fixed absorber in a solar parabolic concentrator, which was a project supported by the Ministry of Education, Science and Technological Development of Republic of Serbia. The device which is used to transform solar energy to heat refers to a solar collector. Solar thermal collectors have been widely used to concentrate solar radiation and convert it into mediumhigh-temperature thermal processes. In addition, the list of possible alternative applications of this technology is growing, due to the problems of oil dependency and global warming. They can be designed as various devices including solar cooker [1], solar hydrogen production [2, 3], and Dish Stirling system of harvest electricity $[4,5]$. The main types of concentrating collectors are parabolic dish, parabolic trough, power tower, a Fresnel collector with mirror or lens, and stationary concentrating collectors. The ideal optical configuration for the solar parabolic thermal concentrator is a parabolic mirror. The parabolic mirror is very expensive to fabricate and its cost escalates rapidly with increase of aperture area. The parabolic mirror can be designed with large number of elementary components known as reflecting petals or facets. Usually reflecting petals are made from glass and their thickness is from 0.7 to $1.0 \mathrm{~mm}$. Traditionally, the optical analysis of radiation concentrators has been carried out by means of computer ray-trace programs. Recently, an interesting analytical solution for the optical performance of parabolic dish reflectors with flat receivers was presented by O'Neill and Hudson [6]. Their method for calculating the optical performance is fast and accurate but assumes that the radiation source is a uniform disk. Saleh Ali et al. [7] have presented a study that aims to develop a 3D static solar concentrator that can be used as a low cost and low energy substitute. Their goal was to design solar concentrator for the production of portable hot water in rural India. They used ray tracing software for evaluation of the optical performance of a static 3D Elliptical Hyperboloid Concentrator (EHC). Optimization of the concentrator profile and geometry is carried out to improve the overall performance of the system. Kaushika and Reddy [8] used satellite dish of $2.405 \mathrm{~m}$ in 
diameter with aluminium frame as a reflector to reduce the weight of the structure and the cost of the solar system. In their solar system the average temperature of water vapor was $300^{\circ} \mathrm{C}$, when the absorber was placed at the focal point. The cost of their system was US\$ 950. El Ouederni et al. [9] were testing parabolic concentrator of $2.2 \mathrm{~m}$ in diameter with reflecting coefficient 0.85 . Average temperature in their system was $380^{\circ} \mathrm{C}$. Rafeeu and Ab Kadir [10] have presented simple exercise in designing, building, and testing small laboratory scale parabolic concentrators. They made two dishes from acrylonitrile butadiene styrene and one from stainless steel. Three experimental models with various geometrical sizes and diameters were used to analyze the effect of geometry on a solar irradiation. Liu et al. [11] presented a procedure to design a facet concentrator for a laboratory scale research on medium-temperature thermal processes. The facet concentrator approximates a parabolic surface with a number of flat square facets supported by a parabolic frame and having two edges perpendicular to the concentrator axis. Authors [12] presented a physical and mathematical model of the new offset type parabolic concentrator and a numerical procedure for predicting its optical performances. Also the process of design and optical ray tracing analysis of a low cost solar concentrator for medium-temperature applications is presented. This study develops and applies a new mathematical model for estimating the intercept factor of the solar concentrator based on its geometry and optical behaviour. Pavlovic et al. [13] developed mathematical model of solar parabolic dish concentrator based on square flat facets applied to polygeneration system. The authors developed optimization algorithm for search of optimal geometric, optical, and cost parameters. They have applied Monte Carlo ray tracing methodology which is used for analysis of the optical performance of the concentrator and to identify the set of geometric concentrator parameters that allow for flux characteristics suitable for medium- and high-temperature applications in trigeneration and polygeneration systems. A 164 -facet concentrator will deliver up to $8.15 \mathrm{~kW}$ of radiative power over $15 \mathrm{~cm}$ radius disk located in the focal plane. Their system had an average concentration ratio exceeding 100 . Hasnat et al. [14] presented two prototype parabolic dishes. Dunn et al. [15] investigated experimental evaluation of ammonia receiver geometries with a $9 \mathrm{~m}^{2}$ dish concentrator. The $20 \mathrm{~m}^{2}$ dish is mirrored with around 2,000 flat mirror tile facets arranged in concentric rings on a parabolic fiber glass support structure. Size of mirror facets is from $5 \mathrm{~cm}$ to $10 \mathrm{~cm}$. Johnston et al. [16] analyzed optical performance of spherical reflecting elements for use with parabolic dish concentrators. This concentrator consists of 54 triangular mirrors. The effective rim angle of the dish is $46^{\circ}$. The 54 units are composed of nine separate panel shapes, each of the shapes is duplicated six times. The focal length of system is $13.1 \mathrm{~m}$.

The decision to make solar parabolic concentrator with 11 petals is based on large number of design concepts that are realized in the world. This concept already proved to be useful in solar techniques, especially in production of heat and electrical energy as well as in trigeneration and polygeneration systems.
The basic idea behind this research is to start with primary concept of solar parabolic concentrator which will generate from 10 to $25 \mathrm{~kW}$ in polygeneration systems. Only with employment of parabolic concentrating systems it is possible to obtain high temperatures in range from $200^{\circ} \mathrm{C}$ to $800^{\circ} \mathrm{C}$ and high optical and thermal efficiency of concentrating solar collectors.

\section{Geometrical Model of the Solar Parabolic Concentrator and Receiver}

The design of the solar parabolic thermal concentrator and operation are presented. Optical design is based on parabolic dish with 11 curvilinear trapezoidal petals. Solar dish concentrators are generally concentrators that concentrate solar energy in a small area known as focal point. Dimensions of reflecting surfaces in solar dish concentrator are determined by desired power at maximum levels of insolation and efficiency of collector conversion.

The ray tracing technique is implemented in a software tool that allows the modelling of the propagation of light in objects of different media. This modelling requires the creation of solid models, either by the same software or by any computer aided design (CAD) software. Once in the optical modelling software, portions of the rays of the light source propagate in the flow of light, in accordance with the properties assigned to the relevant objects, which may be absorption, reflection, transmission, fluorescence, and diffusion. The sources and components of the light rays, adhering to various performance criteria involving the system parameters, result in simulation of the spatial and angular distribution, uniformity, intensity, and spectral characteristics of the system. Mathematical representation of parabolic concentrator is paraboloid that can be represented as a surface obtained by rotating parabola around axis. Mathematical equations for the parabolic dish solar concentrator in Cartesian and cylindrical coordinate systems are defined as

$$
x^{2}+y^{2}=4 f z \quad z=\frac{r^{2}}{4 f},
$$

where are $x$ and $y$ are coordinates in aperture plane and $z$ is distance from vertex measured along the line parallel with the paraboloid axis of symmetry; $f$ is focal length of paraboloid, that is, distance from the vertex to the focus along the paraboloid axis of symmetry. The relationship between the focal length and the diameter of parabolic dish is known as the relative aperture and it defines shape of the paraboloid and position of focal point. The shape of paraboloid can be also defined by rim angle $\psi_{\text {rim }}$. Usually paraboloids that are used in solar collectors have rim angles from 10 degrees up to 90 degrees. The relationship between the relative aperture and the rim angle is given by

$$
\frac{f}{D}=\frac{1}{4 \tan \left(\psi_{\text {rim }} / 2\right)} .
$$

The paraboloid with small rim angles has the focal point and receiver at large distance from the surface of concentrator. 
The paraboloid with rim angle smaller than $50^{\circ}\left(\psi_{\text {rim }}=\right.$ $45,6)$ is used for cavity receivers while paraboloids with large rim angles are most appropriate for the external volumetric receivers (central receiver solar systems). $f / D=0.59 . f / D$ $=0.59$ is focus/diameter ratio-ratios of focal distance to diameter of aperture reflector. Increasing the ratio $f / d$ reduces the rim angle. Paraboloid, with marginal angle, is a little curved, and its focal point and the receiver must be far from the surface of the concentrator. Paraboloid with rim angle of less than $50^{\circ}$ is used when reflective radiation passes into the cavity of the receiver, while the paraboloids with large edge angles are most suitable for external receivers.

The geometric concentration ratio can be defined as the area of the collector aperture $A_{\text {app }}$ divided by the surface area of the receiver $A_{\text {rec }}$ and can be calculated by

$$
\mathrm{CR}_{g}=\left(\sin ^{2} \theta_{a}\right)^{-1}=A_{c} A_{r}{ }^{-1}=\frac{A_{\mathrm{app}}}{A_{\mathrm{rec}}} .
$$

The designed solar parabolic concentrator has geometric concentration ratio $\mathrm{CR}_{g}=100$.

\subsection{Parameters Design of Solar Parabolic Concentrator.} Mechanical design of the solar parabolic concentrator is done in 3D design software CATIA, Dassault Systemes, USA. Parabolic shape of solar concentrator is obtained by entering $x$ and $y$ coordinates for selected points. For calculation of necessary points that define parabola public domain software Parabola Calculator 2.0 [17] is used. The calculated coordinates ( $x$ and $y$ ) for designed parabola are shown in Table 1 . The calculated values are performed for 22 points in the parabola curve. The equation for parabola is

$$
y=\frac{x_{1}^{2}+x_{2}^{2}}{4 f}=\frac{x^{2}}{9040} .
$$

Geometrical model of solar parabolic concentrator is parametrically designed from calculated coordinates and it is shown in Figure 1(a). Selected model of solar dish concentrator with 11 petals requires very precise definition of parameters during geometrical modelling of system. Results obtained by optical analysis of solar concentration system are very much dependent on the selected method of the CAD model generation. Figure 2 represents the rear side of solar parabolic dish collector and geometrical model of construction of paraboloid curve with 11 petals/liefs. The geometrical parameters from Mathematica is exported in CATIA software and have built 3D CAD model.

Figures 3(a) and 3(b) show the profile of spiral absorber tube and whole assembly of solar parabolic dish collector with receiver in their focus.

A truncated paraboloid of revolution (circular paraboloid) is obtained by rotating the parabola segment about its axis (Figure 4). Consider a concentrator consisting of 11 trapezoidal reflective petals of identical nonoverlapping trapezoidal segments. 3D model of trapezoidal reflective petal of solar parabolic concentrator is presented in Figure 4. The outer diameter of corrugated pipe is $D_{e}=12.2 \mathrm{~mm}$, inner diameter is $D_{i}=9.3 \mathrm{~mm}$, and thickness of wall pipes is $s=$ $0.25 \mathrm{~mm}$. Figure 5 shows the paraboloidal lief/petal of solar
TABLE 1: Coordinates of designed parabola.

\begin{tabular}{cccccccc}
\hline$X(\mathrm{~cm})$ & -190.0 & -158.3 & -126.6 & -95.00 & -63.33 & -31.67 & 0.0 \\
$Y(\mathrm{~cm})$ & 39.9 & 27.7 & 17.78 & 9.97 & 4.43 & 1.12 & 0.0 \\
$X(\mathrm{~cm})$ & 31.67 & 63.33 & 95.00 & 126.6 & 158.3 & 190.0 & - \\
$Y(\mathrm{~cm})$ & 1.12 & 4.43 & 9.97 & 17.78 & 27.7 & 39.9 & - \\
\hline
\end{tabular}

TABLE 2: Design parameters of solar parabolic concentrator.

\begin{tabular}{lcc}
\hline Parameters & Numerical value & Unit \\
\hline Aperture radius $R_{1}$ & 0.2 & {$[\mathrm{~m}]$} \\
Radius of smaller hole $R_{2}$ & 1.9 & {$[\mathrm{~m}]$} \\
Gross collector area $A_{\text {gross }}$ & 11.82 & {$\left[\mathrm{~m}^{2}\right]$} \\
$\begin{array}{l}\text { The cross section of the opening } \\
\text { parabola } A_{\text {proj }}\end{array}$ & 9.89 & {$\left[\mathrm{~m}^{2}\right]$} \\
$\begin{array}{lcc}\text { A sheltered area of the concentrator } \\
A_{\text {shadow }}\end{array}$ & 0.126 & {$\left[\mathrm{~m}^{2}\right]$} \\
$\begin{array}{l}\text { The effective area of the } \\
\text { concentrator } A_{\text {ef }}=A_{\text {proj }}-A_{\text {shadow }}\end{array}$ & 9.764 & {$\left[\mathrm{~m}^{2}\right]$} \\
Receiver diameter & 0.40 & {$[\mathrm{~m}]$} \\
& Directly irradiated & \\
Shape of receiver & corrugated spiral & - \\
& thermal absorber pipe & \\
Depth of the concentrator & 0.399 & {$[\mathrm{~m}]$} \\
Focal length & 2.26 & {$[\mathrm{~m}]$} \\
$\psi_{1}$ & 6 & {$\left[{ }^{\circ}\right]$} \\
$\psi_{2}$ & 45.6 & {$\left[{ }^{\circ}\right]$} \\
\hline
\end{tabular}

paraboloidal concentrator system. The front side of the petals is reflective surface with coefficient of reflectance of $90 \%$ (mirrored specular reflectance surface).

$R_{1}$ is aperture radius of absorber, while $R_{2}$ is aperture radius of solar dish concentrator/reflector. The small hole is constructive approach.

Detailed design parameters of solar parabolic concentrator are given in Table 2 .

Receiver-absorber is placed in focal area where reflected radiation from solar concentrator is collected. In the process of designing parabolic solar concentrators one always seeks for the minimum size of the receiver. With small receiver size one can reduce heat losses as well as cost of whole system. Also small receiver size provides increase of absorbed flux on the surface of receiver. This is the way of obtaining greater efficiency in conversion of solar radiation to heat. In our system receiver-absorber is Archimedean spiral corrugated pipe type with diameter of $400 \mathrm{~mm}$. It is shown in Figure 6.

In this paper only optical properties of receiver are analyzed. In our further research we plan to model all necessary details of receiver's geometry which are important for conversion of solar energy into heat of fluid that is used for transfer energy. 


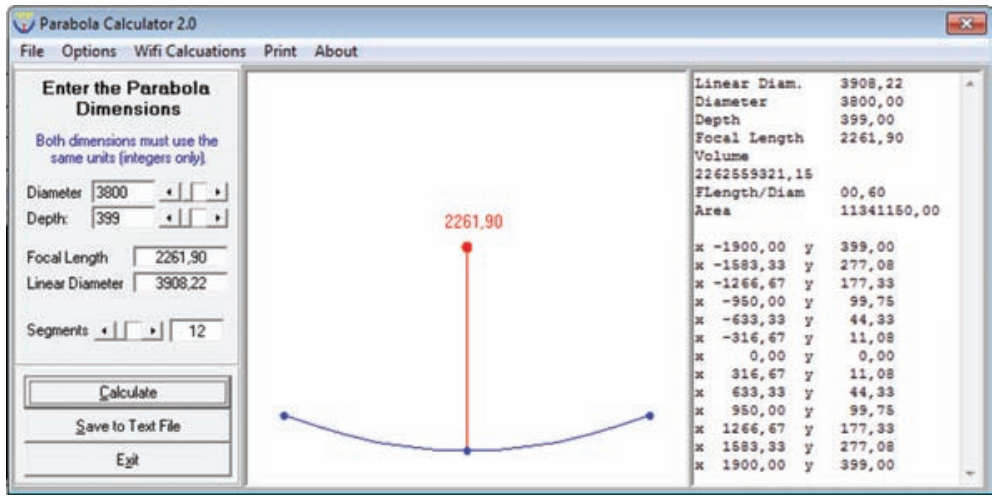

(a)

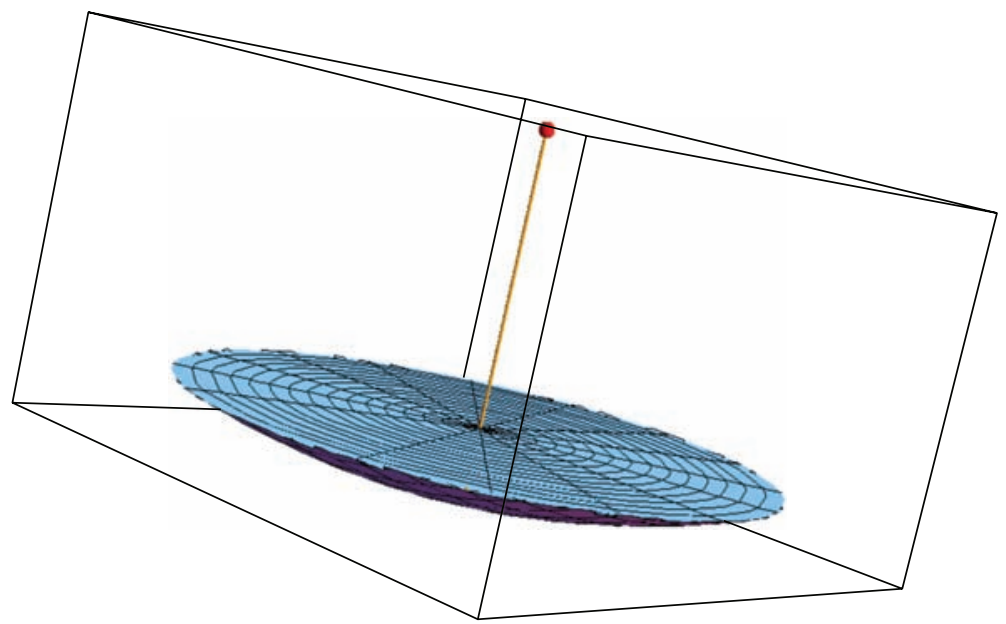

(b)

Figure 1: (a) Parabola Calculator 2.0 [17]. (b) Paraboloid generated from Mathematica Wolfram software.
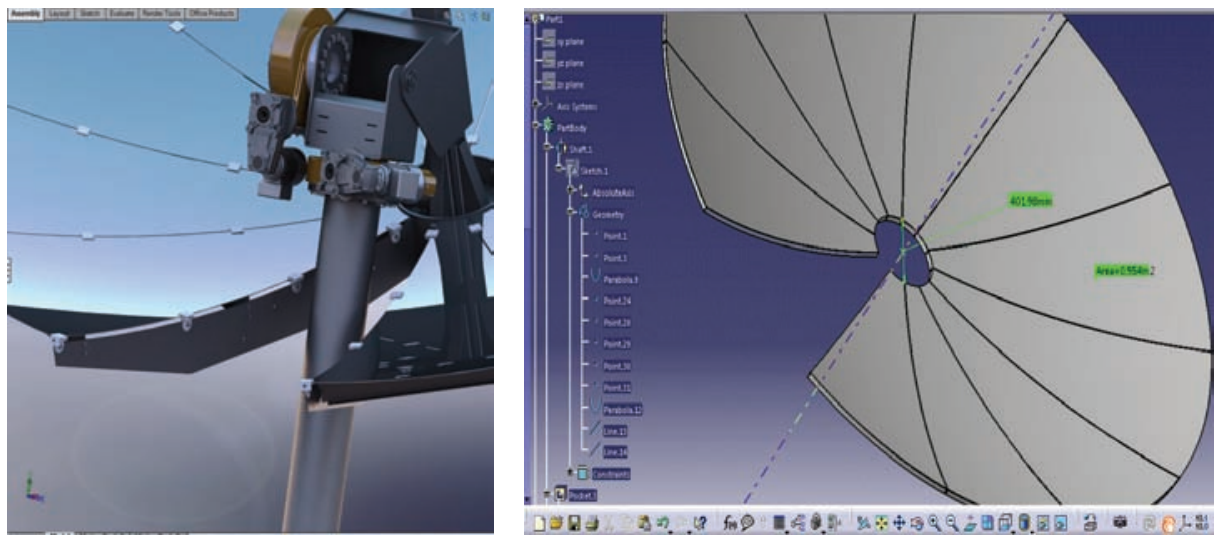

FIGURE 2: Mirrored segmented parabolic dish concentrator.

\section{Numerical Simulations and Ray Tracing Studies to Determine the Optical Characteristics of the Solar Image}

For optical ray tracing analysis of solar parabolic thermal concentrator software TracePro, Lamda Research Corporation, USA, is used. Simulations have been performed using the technique of ray tracing to describe the behaviour of parabolic dish solar concentrators and such simulations have been used to study the behaviour of such concentrators in nonimaging optics; however, there is no such study or simulation of ray tracing type to describe parabolic solar concentrators. In TracePro all material properties are assigned. 11 trapezoidal reflective petals are defined 


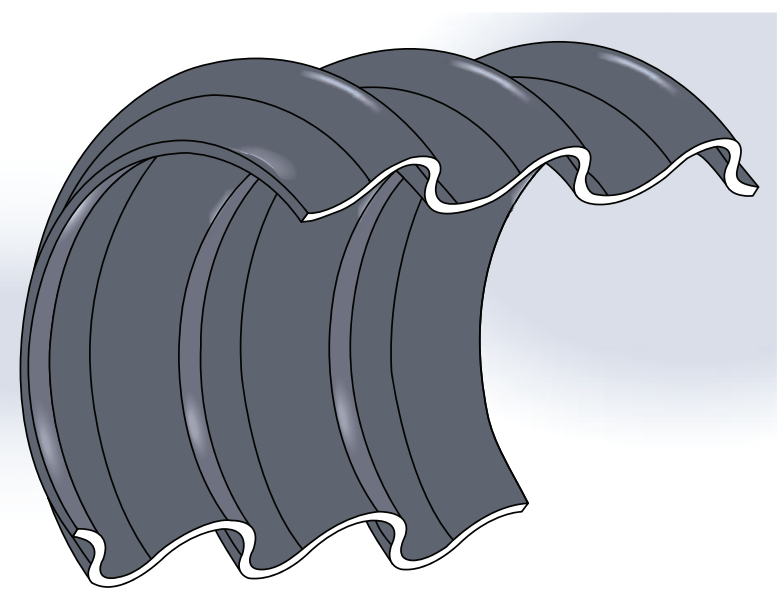

(a)

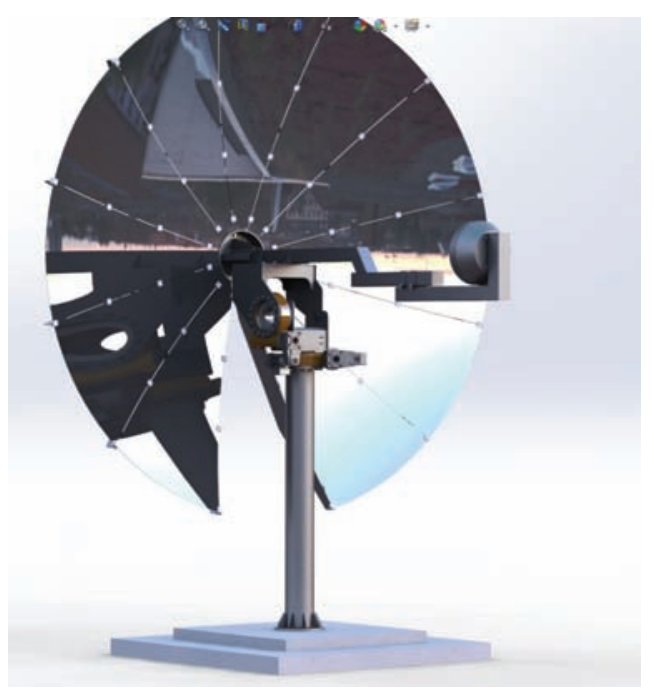

(b)

FIGURE 3: 3D CAD model of thermal solar concentration system and sinusoidal profile of corrugated pipe of spiral absorber.

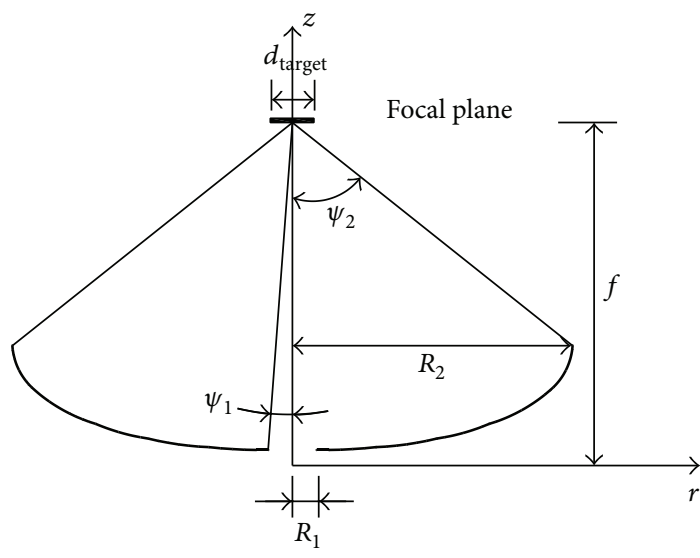

FIGURE 4: Schematic of truncated parabola.

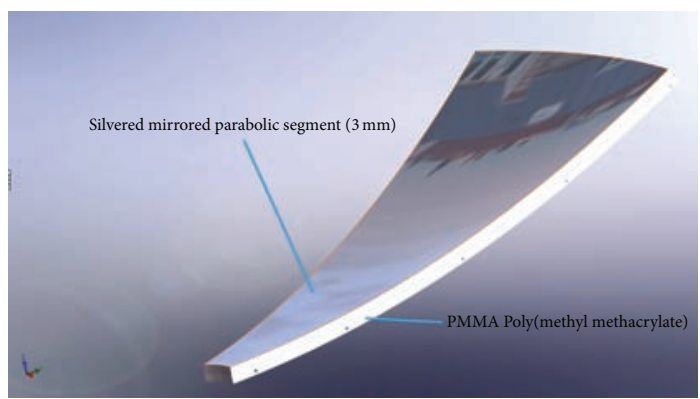

FIGURE 5: Trapezoidal reflective petal of solar parabolic concentrator.

as standard mirrors with reflective coating. Reflection coefficient was 95\%. Receiver was cylinder with diameter $400 \mathrm{~mm}$ placed on $2075 \mathrm{~mm}$ from vertex of parabolic dish (optimal focal distance from vertex of parabolic solar concentrator). Absorbing surface was defined as perfect

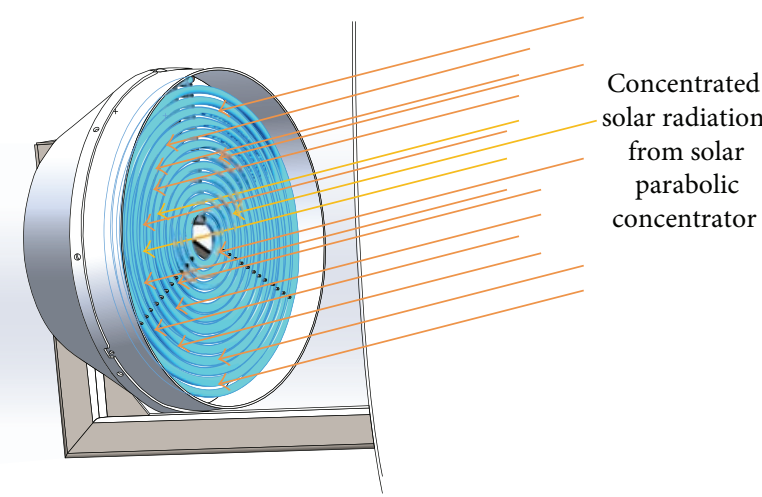

FIGURE 6: Solar cavity receiver-spiral corrugated pipe type of solar absorber.

absorber. After definition of geometry of solar parabolic concentrator radiation source was defined. Radiation source was circular with diameter the same as diameter of parabolic dish $(3800 \mathrm{~mm})$. Radiation source was placed $2000 \mathrm{~mm}$ from vertex of parabolic dish and had circular grid pattern for generating 119401 rays for Monte Carlo ray tracing. Spatial profile of generated rays was uniform and angular profile was solar radiation. Input parameter for optical analysis is solar irradiance $800 \mathrm{~W} / \mathrm{m}^{2}$. Experiential value for solar irradiation for town of Niš in Serbia is between $750 \mathrm{~W} / \mathrm{m}^{2}$ and $900 \mathrm{~W} / \mathrm{m}^{2}$. Optical system for solar parabolic concentrator with traced rays is given in Figure 7.

Temperature of the spiral absorber pipe-stagnation surface temperature is about $680^{\circ} \mathrm{C}$. Detailed heat transfer analysis of this solar spiral heat pipe absorber will be presented in my other future papers.

Optical analysis is done by generating and calculating Monte Carlo ray trace for 119401 ray. From all emitted rays only 103029 rays reached absorber surface of which $82 \%$ of 


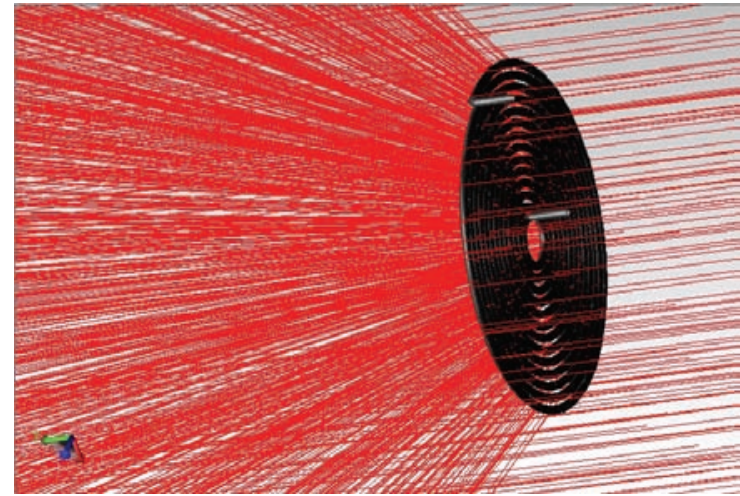

(a)

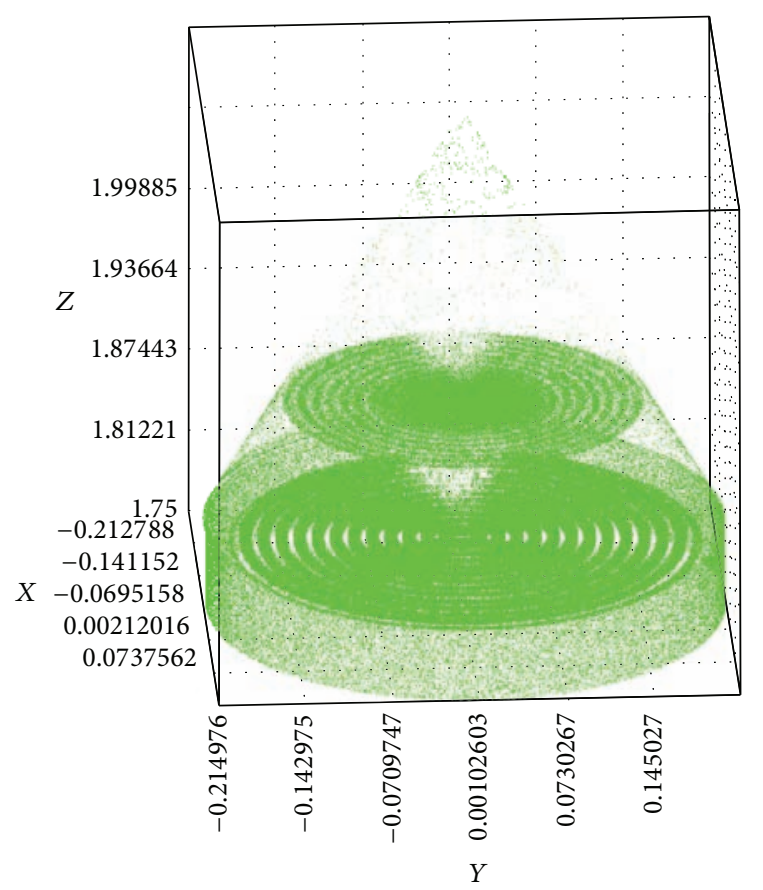

(b)

FIGURE 7: (a) Ray tracing model of solar parabolic concentrator with traced rays. (b) Ray tracing model of solar spiral heat pipe with housing, SolTrace optical software tool, NREL, USA.

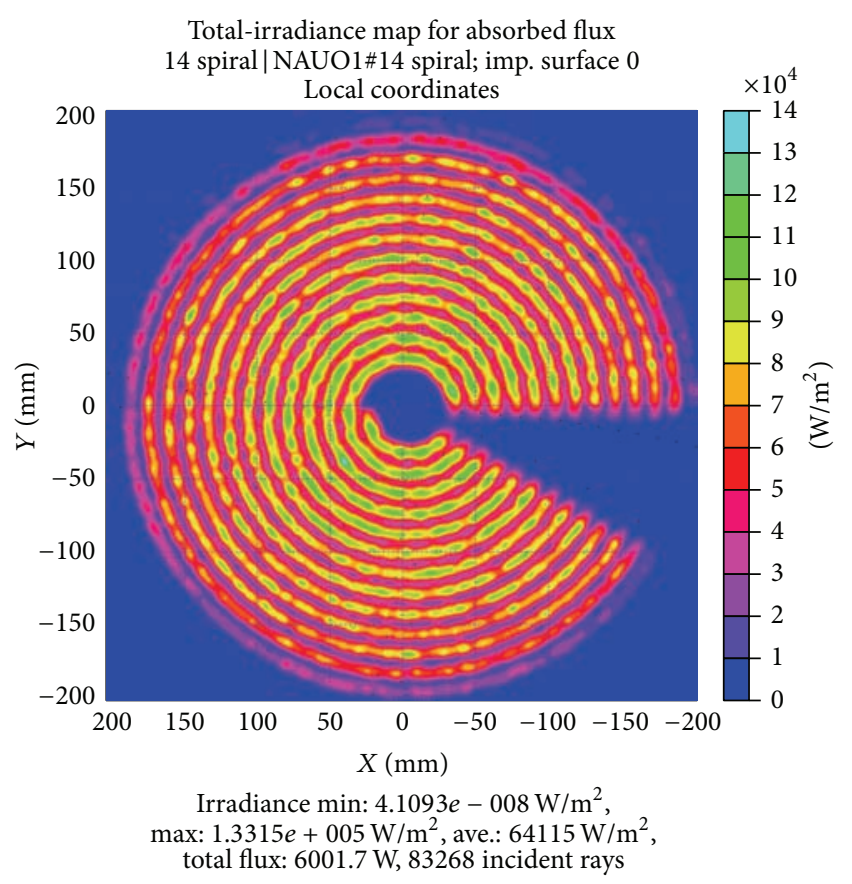

FIGURE 8: Irradiance map for absorbed flux on spiral receiver (solar focal image).

emitted rays are absorbed on receiver. Calculated irradiance for absorbed rays on receiver is from $4.109 \cdot 10^{-8} \mathrm{~W} / \mathrm{m}^{2}$ to $1.331 \cdot 10^{5} \mathrm{~W} / \mathrm{m}^{2}$. Total calculated flux on receiver was
6001.7 W. In Figure 8 total irradiance map for absorbed flux on receiver is shown. When the sunlight shines on the solar collector including the direct and scattered radiation, there are three conditions affecting the absorption properties of the solar collector: (1) direct solar radiation absorbed directly by the solar collector and the light energy after the specular reflection; (2) the light energy of the scatter solar radiation after the diffuse and specular reflection; (3) the light energy of the direct solar radiation after the diffuse reflection. The slope of incident light is different in different latitude and time.

Figure 9 represents path sun rays from the source to the concentration of absorbing surface corrugated profile of absorber tube receiver.

We take city of Nis (Laboratory for Solar Thermal Engineering) as an example. Nis is located in southeast Serbia longitude $43.30^{\circ}$ north latitude and $21.90^{\circ}$ east longitude. Solar Parabolic Dish concentrator (Figure 9(b)) is tracking the sun with motorized slewing drive solar tracking systems in two axes: azimuth and elevation (dual axis solar tracking precision system).

The influences of absorption rate for the sinusoidal corrugated absorber plate by aspect ratio and the slope of incident light are plotted in Figure 10. From the optical point of view, the time of light reflection absorption increases with the aspect ratio, which causes the absorption rate of the absorber plate to increase. Particularly, the absorption rate is close to 1 , when the aspect ratio tends to infinity. 


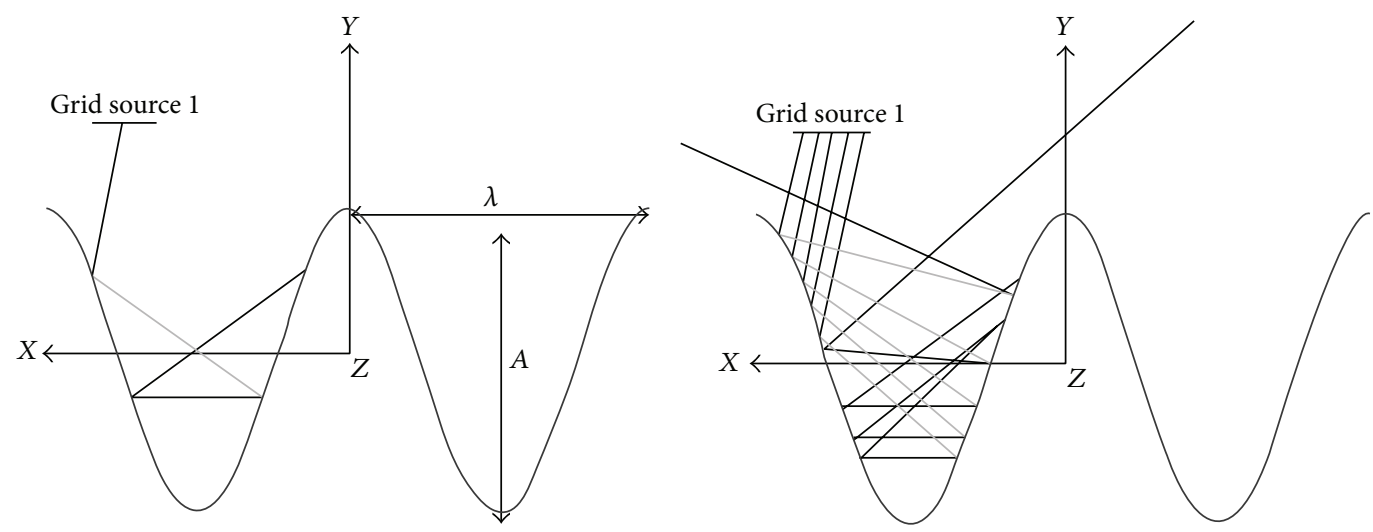

(a)

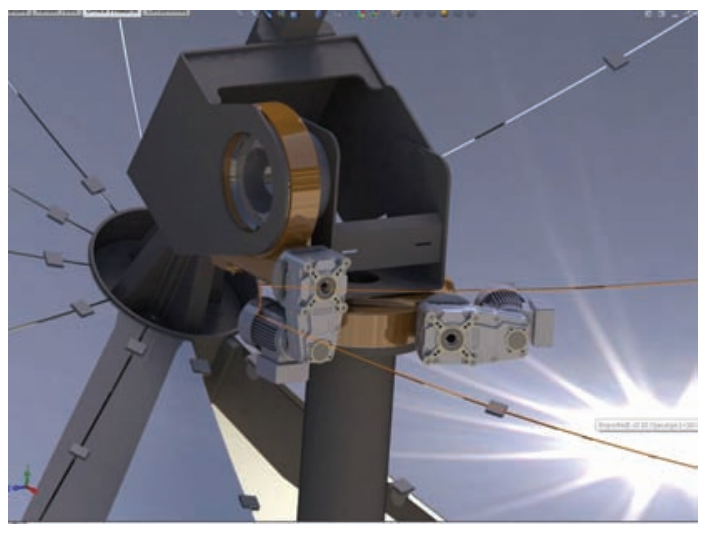

(b)

FIgURE 9: (a) Schematic diagram of optical path shinning on the sinusoidal corrugated pipe absorber. (b) Dual axis solar tracking system with slew drives and gear motor boxes.

Figure 10 right shows ray tracing model, radiation control volume grid (1000 elements) for part of corrugated pipe.

Figure 10 shows control volume grid (1000 elements) for part of corrugated pipe, small part from all spiral corrugated absorber; this figure presented incident solar flux in control volume-small control volume-3 wrinkles-folds.

According to the boundary conditions, the optical properties can be obtained after many simulation calculations, which is called the ray tracing method [11].

Volume radiation flux distribution (incident and absorbed flux at the wall surface of profiles), software TracePro generated this bmp. file.

Figure 11 shows distribution of incident and absorbed solar hear flux at the corrugated surfaces of spiral thermal absorber.

Figure 12 shows scattered model for reflection of incidence angle for solar parabolic dish concentrator with 11 curvilinear trapezoidal reflective petals. In general, the reflectance of a surface increases with angle of incidence. But for a mirror, the reflectance is already very high when $\mathrm{AOI}=$ $0^{\circ}$. It cannot increase much more. This is why the peak BRDF does not change with AOI. However, the reflectance of a black surface increases with AOI, no matter how black the surface is.

\section{Conclusions}

This paper presents optical analysis of the solar parabolic concentrator using the ray tracing software TracePro. One can see that results obtained from optical design of solar parabolic concentrator are satisfactory. Total flux in focal area is good. Irradiance distribution for absorbed flux is relatively uniform for small area for absorber. A detailed simulation and analysis were conducted to evaluate the absorption rate of sinusoidal corrugated absorber pipe. As a next step, various analysis and simulations of the model are considered. Among others are variation of number of petals, size of petals, and shape of petals. Optimization methodology is not the topic of this work. Here it is only shown that the application of the Monte Carlo method can be performed to determine optimal parameters for obtaining the maximum value of total solar flux density and average irradiance-solar radiant energy at the spiral corrugated absorber.

The significance of this work is primarily in finding the optimal parameters of the geometric and optical parameters in the concentrator and receiver/absorber of concentration radiation. The main objective of this work is to find the optimal parameters in order to develop a prototype of a solar parabolic concentrator aspects of polygeneration systems, 

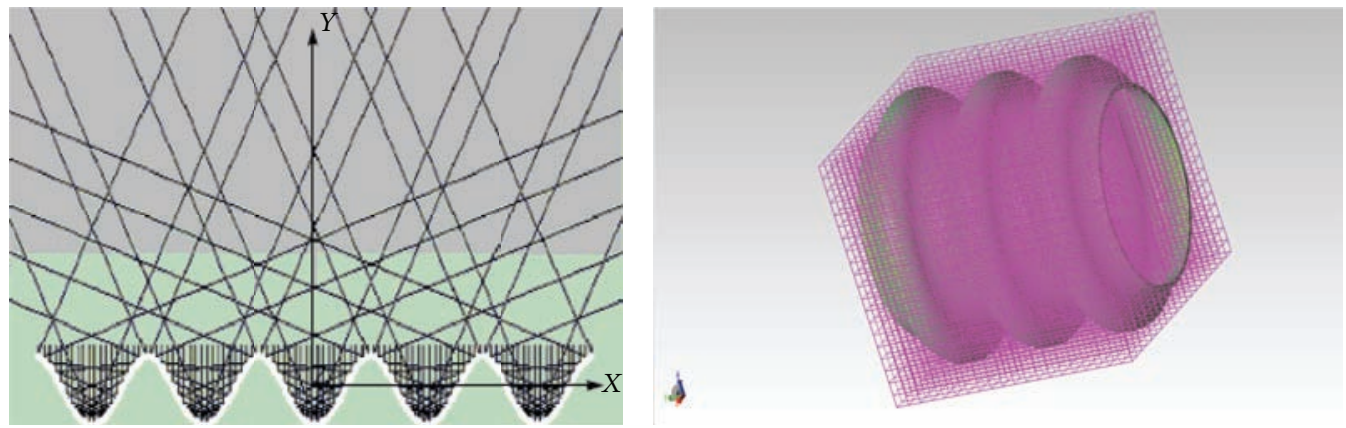

(a)

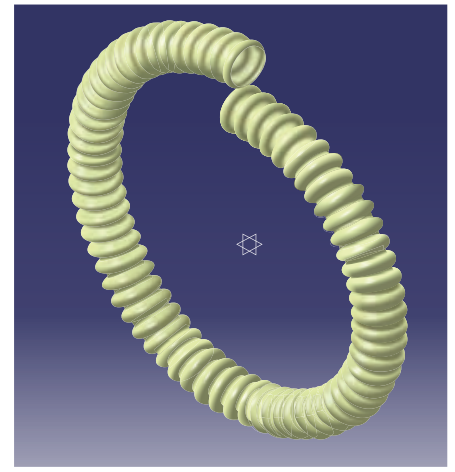

\begin{tabular}{|c|c|c|c|c|c|c|c|c|}
\hline DN & $\begin{array}{l}\text { Codice tubo } \\
\text { (tube code) }\end{array}$ & $\begin{array}{c}\text { Fil. } \\
\text { connessione } \\
\text { (thread } \\
\text { connection) }\end{array}$ & $\begin{array}{c}D_{i} \\
(\mathrm{~mm})\end{array}$ & $\begin{array}{c}D_{e} \\
(\mathrm{~mm})\end{array}$ & $\begin{array}{c}\text { Spessore } \\
\text { (thickness) } \\
\text { S (nm) }\end{array}$ & $\begin{array}{l}\text { Superfice lineica } \\
\text { interna (inner linear } \\
\text { surface) }\left(\mathrm{m}^{2} / \mathrm{m}\right)\end{array}$ & $\begin{array}{l}\text { Superfice lineica } \\
\text { esterna (outer linear } \\
\text { surface) }\left(\mathrm{m}^{2} / \mathrm{m}\right)\end{array}$ & $\begin{array}{c}\text { Volume lineico } \\
\text { (linear volume) } \\
(\mathrm{L} / \mathrm{m})\end{array}$ \\
\hline 10 & TFA38 & $3 / 8^{\prime \prime}$ & 9.3 & 12.2 & 0.25 & 0.0407 & 0.0429 & 0.0890 \\
\hline 12 & TFA12 & $1 / 2^{\prime \prime}$ & 13.2 & 16.8 & 0.3 & 0.0565 & 0.0591 & 0.1730 \\
\hline 12 & TFG12N & $1 / 2^{\prime \prime}$ & 12.0 & 15.8 & 0.3 & 0.0540 & 0.0568 & 0.1500 \\
\hline 157 & TFA34-TFG15N & $3 / 4^{\prime \prime}$ & 15.8 & 20.0 & 0.3 & 0.0702 & 0.0730 & 0.2480 \\
\hline 207 & TFA25-TFG20N & $1^{\prime \prime}$ & 19.7 & 25.0 & 0.3 & 0.0912 & 0.0942 & 0.3830 \\
\hline 257 & TFA32-TFG25N & $11 / 4^{\prime \prime}$ & 26.5 & 33.0 & 0.3 & 0.1313 & 0.1345 & 0.7000 \\
\hline 32 & TFA40 & $11 / 2^{\prime \prime}$ & 33.0 & 41.0 & 0.35 & 0.1757 & 0.1799 & 1.0464 \\
\hline
\end{tabular}

(b)

FIGURE 10: (a) Ray tracing and radiation control volume grid (1000 elements) for part of corrugated pipe. (b) Spiral corrugated one coil heat absorber from total 13 coils.
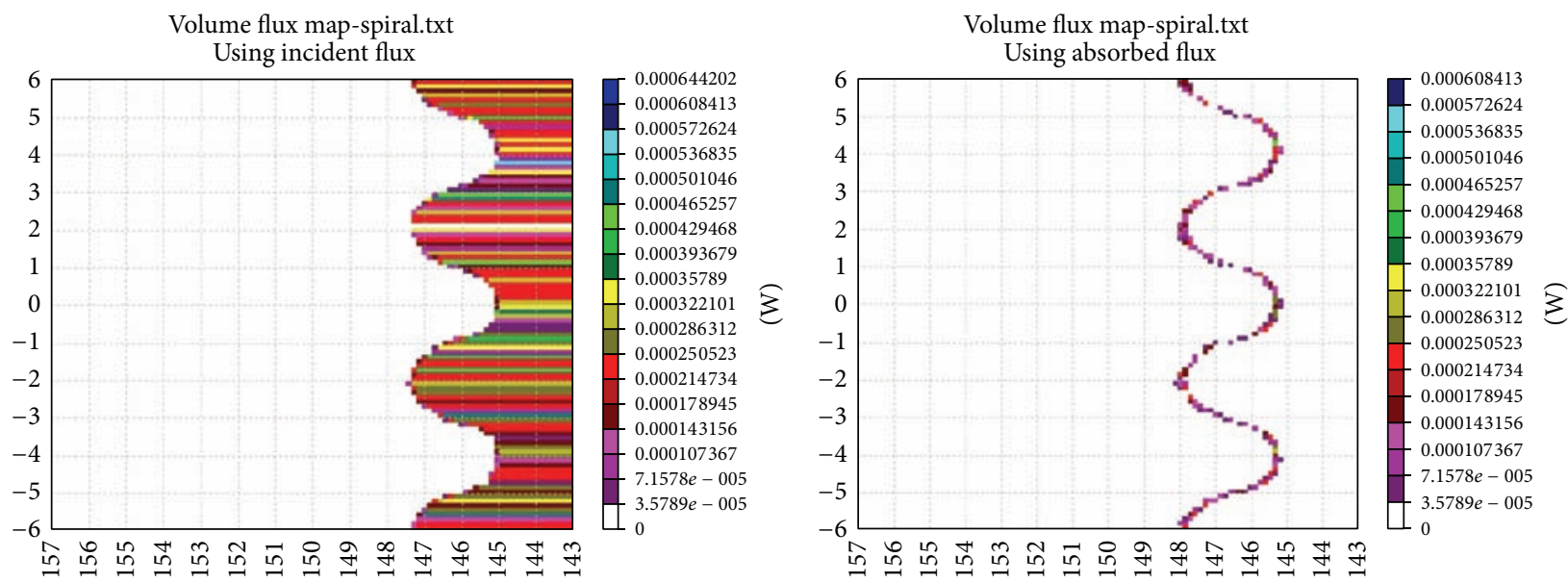

FIGURE 11: Volume radiation flux distribution (incident and absorbed flux).

production of heating, cooling, and electricity using solar energy in Southeastern Europe.

\section{Nomenclature}

Latin Symbols
A: Area, in $[\mathrm{m}]$
a: Facets height, in $[\mathrm{m}]$

$x: \quad$ Coordinate at the $X$ direction

$y$ : Coordinate at the $Y$ direction

$z$ : $\quad$ Coordinate at the $Z$ direction

$A_{\mathrm{pp}}: \quad$ Collector $s$ aperture surface $\left[\mathrm{m}^{2}\right]$

$A_{r}: \quad$ Receiver aperture surface $\left[\mathrm{m}^{2}\right]$

$d_{\text {target }}$ : Diameter of receiver surface $[\mathrm{m}]$

$\mathrm{CR}_{g}$ : Average geometric concentration ratio [-]

$f: \quad$ Focal length $[\mathrm{m}]$ 


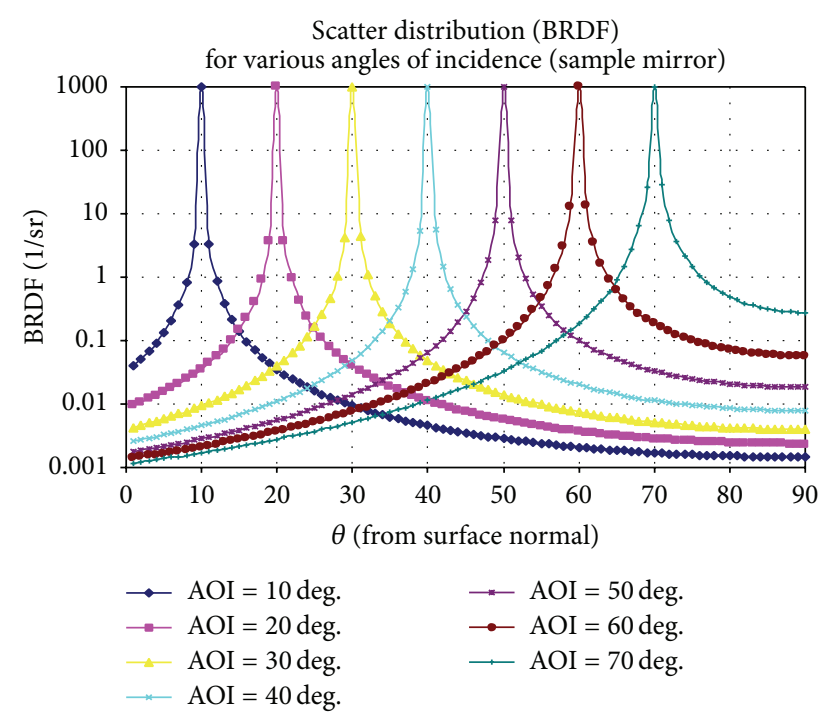

FIgURE 12: $\mathrm{ABg}$ giving the BRDF as a function of incidence angle.

$G_{0}: \quad$ Incident direct solar flux, in $\left[\mathrm{Wm}^{-2}\right]$

I: $\quad$ Total radiative intensity, in $\left[\mathrm{Wm}^{-2} \mathrm{sr}^{-1}\right]$

$\dot{Q}: \quad$ Radiative power, in $[\mathrm{kW}]$

$\dot{q}: \quad$ Radiative flux, in $\left[\mathrm{Wm}^{-2}\right]$

$\dot{\dot{q}}: \quad$ Average radiative flux, in $\left[\mathrm{Wm}^{-2}\right]$

$R, r:$ Radius, in $[\mathrm{m}]$

$\vec{r}: \quad$ Position vector $[-]$

$\eta_{c}: \quad$ Collection efficiency $[-]$

$\theta: \quad$ Incident angle, in $\left[^{\circ}\right]$

$\psi_{\text {rim }}:$ Rim angle, in $\left[{ }^{\circ}\right]$

$\phi, \varphi$ : Azimuthal angle, in $\left[{ }^{\circ}\right]$

$\gamma: \quad$ Constant.

\section{Greek Symbols}

$\beta$ : Facet tilt angle, rad; limb darkening parameter

$\delta, \varepsilon$ : Small positive number

$\rho$ : Density of the fluid, in $\left[\mathrm{kg} \mathrm{m}^{-3}\right]$.

\section{Subscripts}

cs: Circumsolar

ffc: Flat-facet concentrator

sun: Corresponding to sun disk.

\section{Conflict of Interests}

The authors declare that there is no conflict of interests regarding the publication of this paper.

\section{Acknowledgments}

This paper is done within the research framework of research projects: III42006: Research and Development of Energy and Environmentally Highly Effective Polygeneration Systems Based on Renewable Energy Resources. Project is financed by Ministry of Education, Science and Technological Development of Republic of Serbia. The authors would like to acknowledge the help provided by the Lambda Research Corporation by allowing the use of the TracePro software for the Ph.D. research of Saša R. Pavlovic.

\section{References}

[1] A. A. Badran, I. A. Yousef, N. K. Joudeh, R. A. Hamad, H. Halawa, and H. K. Hassouneh, "Portable solar cooker and water heater," Energy Conversion and Management, vol. 51, no. 8, pp. 1605-1609, 2010.

[2] A. S. Joshi, I. Dincer, and B. V. Reddy, "Solar hydrogen production: a comparative performance assessment," International Journal of Hydrogen Energy, vol. 36, no. 17, pp. 11246-11257, 2011.

[3] P. Furler, J. R. Scheffe, and A. Steinfeld, "Syngas production by simultaneous splitting of $\mathrm{H}_{2} \mathrm{O}$ and $\mathrm{CO}_{2}$ via ceria redox reactions in a high-temperature solar reactor," Energy \& Environmental Science, vol. 5, no. 3, pp. 6098-6103, 2012.

[4] T. Mancini, P. Heller, B. Butler et al., "Dish-stirling systems: an overview of development and status," Journal of Solar Energy Engineering, vol. 125, no. 2, pp. 135-151, 2003.

[5] D. Mills, "Advances in solar thermal electricity technology," Solar Energy, vol. 76, no. 1-3, pp. 19-31, 2004.

[6] M. J. O’Neill and S. L. Hudson, “Optical analysis of paraboloidal solar concentrators," in Proceedings of the Annual Meeting, U.S. Section of International Solar Energy Society, vol. 2.1, pp. 855862, Denver, Colo, USA, August 1978.

[7] I. M. Saleh Ali, T. S. O’Donovan, K. S. Reddy, and T. K. Mallick, "An optical analysis of a static 3-D solar concentrator," Solar Energy, vol. 88, pp. 57-70, 2013.

[8] N. D. Kaushika and K. S. Reddy, "Performance of a low cost solar paraboloidal dish steam generating system," Energy Conversion \& Management, vol. 41, no. 7, pp. 713-726, 2000.

[9] A. R. El Ouederni, M. Ben Salah, F. Askri, and F. Aloui, "Experimental study of a parabolic solar concentrator," Revue des Energies Renouvelables, vol. 12, no. 3, pp. 395-404, 2009.

[10] Y. Rafeeu and M. Z. A. Ab Kadir, "Thermal performance of parabolic concentrators under Malaysian environment: a case study," Renewable and Sustainable Energy Reviews, vol. 16, no. 6, pp. 3826-3835, 2012.

[11] Z. Liu, J. Lapp, and W. Lipiński, "Optical design of a flat-facet solar concentrator," Solar Energy, vol. 86, no. 6, pp. 1962-1966, 2012.

[12] S. Pavlovic, D. Vasiljevic, V. Stefanovic, Z. Stamenkovic, and S. Ayed, "Optical model and numerical simulation of the new offset type parabolic concentrator with two types of solar receivers," Facta Universitatis, Series: Mechanical Engineering, vol. 13, no. 2, pp. 169-180, UDC 535.2, 2015.

[13] S. R. Pavlovic, V. P. Stefanović, and S. H. Suljković, "Optical modeling of a solar dish thermal concentrator based on square flat facets," Thermal Science, vol. 18, no. 3, pp. 989-998, 2014.

[14] A. Hasnat, P. Ahmed, M. Rahman, and K. A. Khan, "Numerical analysis for thermal design of a paraboloidal solar concentrating collector," International Journal of Natural Sciences, vol. 1, no. 3, pp. 68-74, 2011.

[15] R. Dunn, K. Lovegrove, G. Burgess, and J. Pye, "An experimental study of ammonia receiver geometries for dish concentrators," Journal of Solar Energy Engineering-Transactions of the ASME, vol. 134, no. 4, Article ID 041007, 2012. 
[16] G. Johnston, K. Lovegrove, and A. Luzzi, “Optical performance of spherical reflecting elements for use with paraboloidal dish concentrators," Solar Energy, vol. 74, no. 2, pp. 133-140, 2003.

[17] http://mscir.tripod.com/parabola/. 


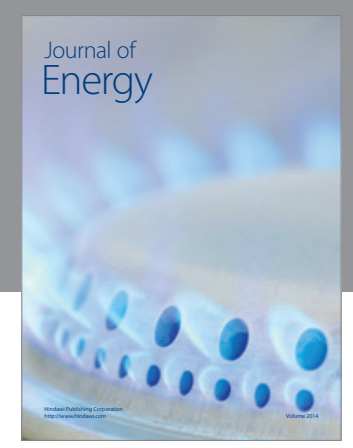

Journal of

Industrial Engineering
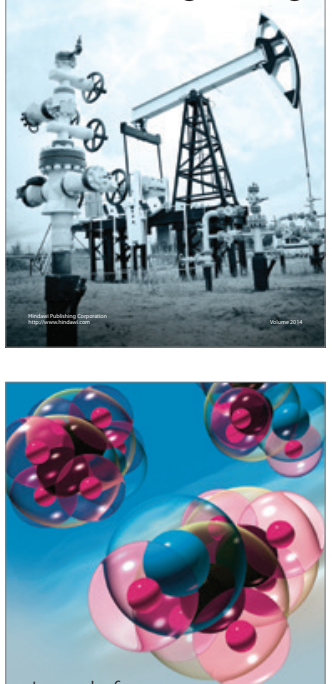

Fuels
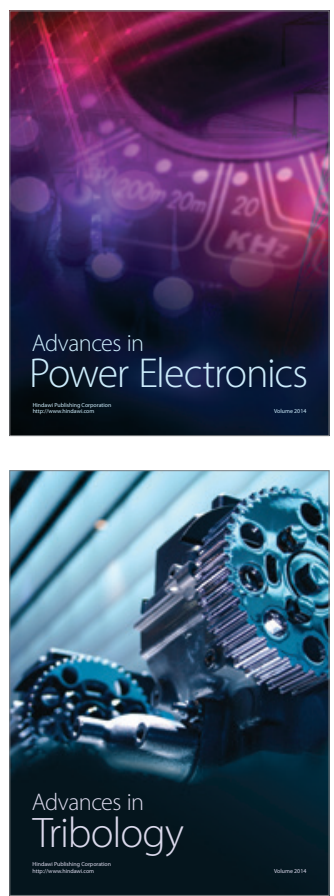

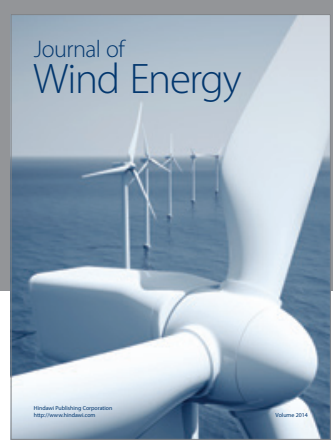

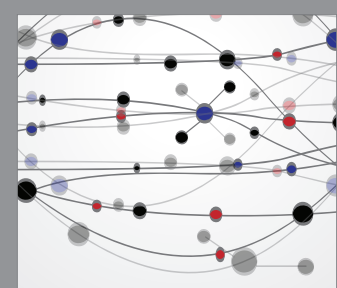

The Scientific World Journal

Submit your manuscripts at http://www.hindawi.com

Journal of

Structures
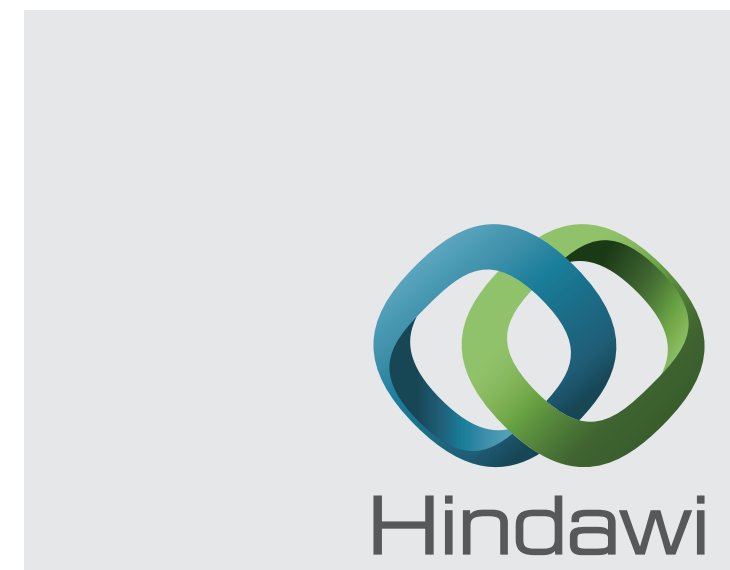

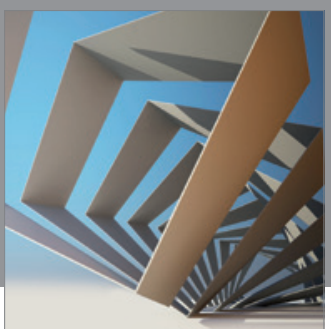

Rotating

Machinery
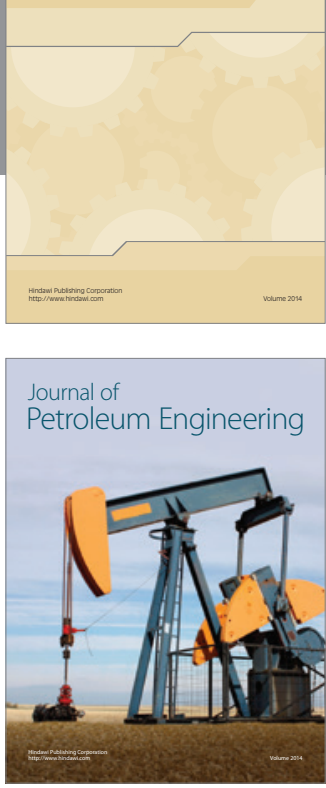

Journal of

Solar Energy
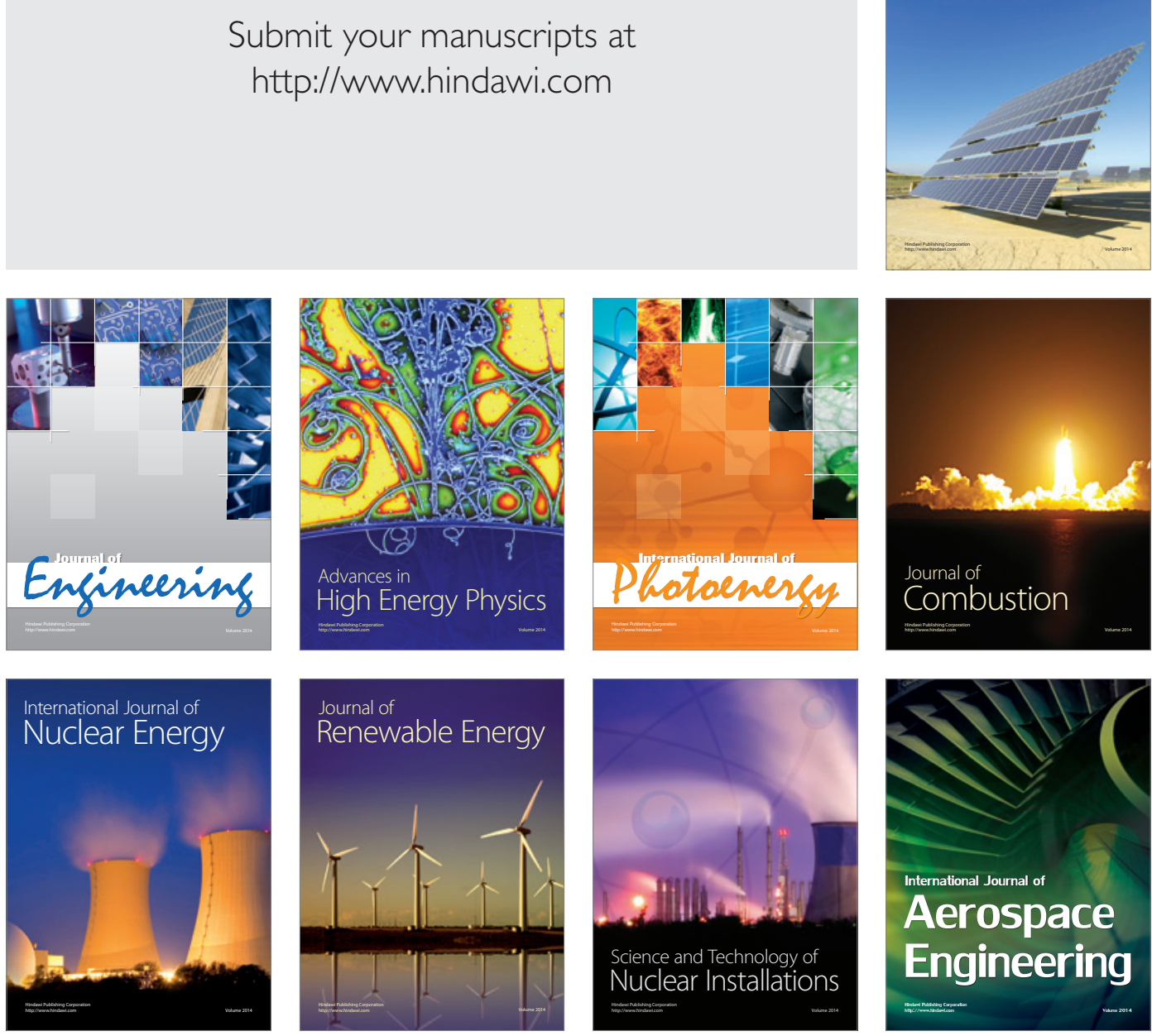